\title{
Color-luminance relationships and the McCollough effect
}

\author{
MICHAEL A. WEBSTER and GOKHAN MALKOC \\ University of Nevada, Reno, Nevada
}

\begin{abstract}
The McCollough effect is an orientation-specific color aftereffect induced by adapting to colored gratings. We examined how the McCollough effect depends on the relationships between color and luminance within the inducing and test gratings and compared the aftereffects to the color changes predicted from selective adaptation to different color-luminance combinations. Our results suggest that the important contingency underlying the McCollough effect is between orientation and colorluminance direction and are consistent with sensitivity changes within mechanisms tuned to specific color-luminance directions. Aftereffects are similar in magnitude for adapting color pairs that differ only in S cone excitation or $\mathrm{L}$ and $\mathrm{M}$ cone excitation, and they have a similar dependence on spatial frequency. In particular, orientation-specific aftereffects are induced for S cone colors even when the grating frequencies are above the $\mathrm{S}$ cone resolution limit. Thus, the McCollough effect persists even when different cone classes encode the orientation and color of the gratings.
\end{abstract}

In a well-known study, McCollough (1965) described a color aftereffect that is contingent on pattern orientation. For example, after the viewing of a vertical grating of green and black bars alternated in time with a horizontal grating of red and black bars, a vertical achromatic grating appears reddish while a horizontal achromatic grating appears greenish. The colors perceived in the test gratings are thus specific to the orientation of the inducing gratings and are complementary to the inducing gratings' colors. The contingent adaptation between form and color revealed by the McCollough effect (ME) generated wide interest, and the effect continues to be extensively studied. However, the processes underlying the aftereffect are still uncertain. For example, it is unclear to what extent the aftereffect reflects an example of general perceptual adjustments versus adjustments that may be specialized for encoding specific stimuli, and whether these adjustments are more closely related to sensory adaptation or learning (Barlow, 1990; Dodwell \& Humphrey, 1990; Harris, 1980; Siegel \& Allan, 1992; Stromeyer, 1978).

Most models of the ME have emphasized the pairings between orientation and color in the stimuli. Here, we focus instead on the pairings between luminance contrast and color. Several previous studies have found that conventional MEs are not generated when the inducing gratings are equiluminant (e.g., when the inducing gratings are composed of red and gray or green and gray stripes that have the same luminance, and the test gratings are composed of bright and dark stripes that are both achromatic;

This research was supported by NIH Grant EY10834. The authors thank J. D. Mollon for discussions and Celeste McCollough Howard, Charles F. Stromeyer III, and Osvaldo Da Pos for very helpful comments on the manuscript. Correspondence should be addressed to $\mathrm{M}$. A. Webster, Department of Psychology/296, University of Nevada, Reno, NV 89557 (e-mail: mwebster@scs.unr.edu).
Allan, Siegel, Kulatunga-Moruzi, Eissenberg, \& Chapman, 1997; Ellis, 1977; Mikaelian, 1980; Stromeyer \& Dawson, 1978). Such results demonstrate that luminance contrast is an important stimulus component for generating the aftereffect (when measured in achromatic test gratings). The absence of the aftereffect at equiluminance has been treated as an anomaly of spatial processing within the chromatic pathways (Savoy, 1987) or as a form of selective associability (Allan et al., 1997). However, Webster (1996) suggested that this result is consistent with the changes in color appearance induced by color contrast adaptation. In studies of contrast adaptation, observers are adapted to a uniform field that slowly flickers between two colors or luminances over time or to a pattern, such as a grating, that varies in color or luminance over space (Webster, 1996). Adapting to a stimulus that varies in time or space along any single color-luminance axis reduces perceived contrast along that axis (Webster \& Mollon, 1991, 1994). This selective sensitivity loss causes all other color-luminance axes to appear biased in direction away from the adapting color axis. For example, Figure 1 shows the changes in perceived color and brightness of test stimuli following adaptation to a uniform field that flickered between bright red and dark green or between bright green and dark red. In this figure, the horizontal axis represents the reddish-greenish variations ( $\mathrm{L}-\mathrm{M}$ chromatic contrast) in the test, whereas the vertical axis represents variations in brightness (luminance contrast). The data plotted are from the study of Webster and Mollon (1994). Adapting to the bright-red/dark-green axis causes pure achromatic increments ("bright grays") to appear greenish and pure achromatic decrements ("dark grays") to appear reddish (unfilled triangles). Concurrently, the bright-red/dark-green adaptation causes equiluminant reds to appear darker and equiluminant greens to appear brighter. Opposite aftereffects are in- 

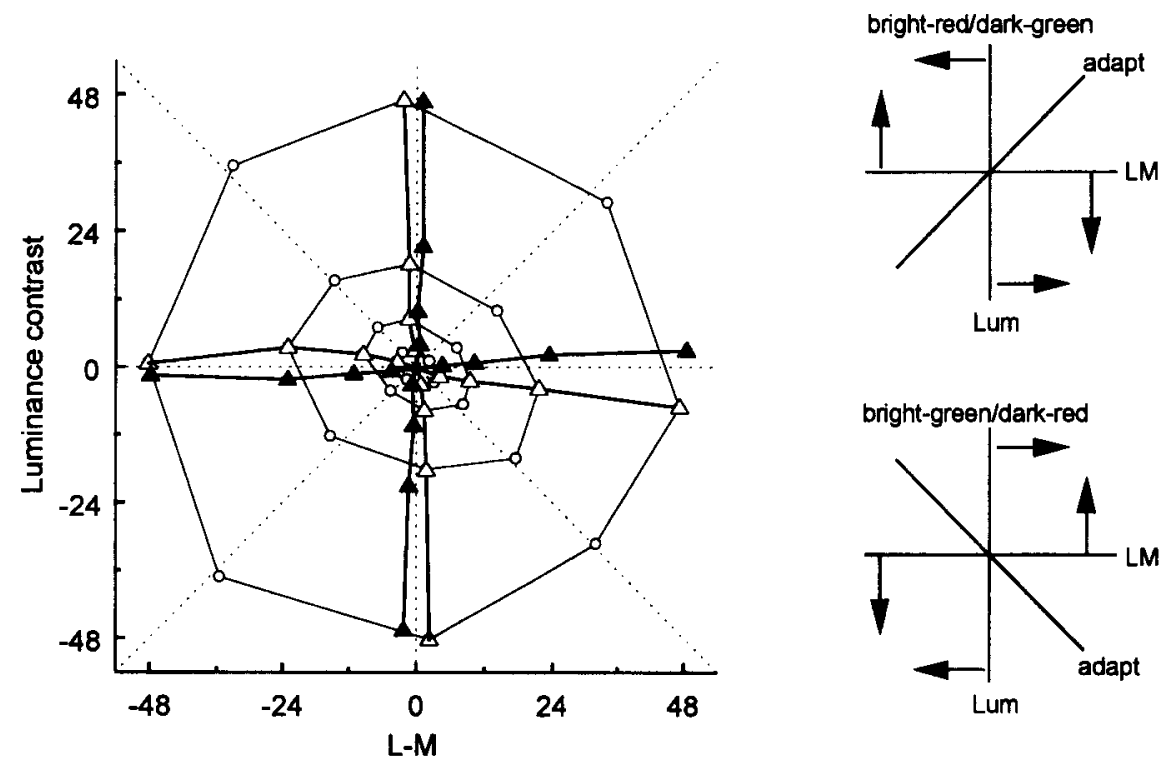

Figure 1. Changes in perceived color and lightness following adaptation to color-luminance modulations in a uniform field (from Webster \& Mollon, 1994). Test stimuli had contrasts of $6,12,24$, or 48 units within the luminance versus $L-M$ plane. Adaptation to a bright-red/darkgreen modulation (along the positive diagonal) reduces sensitivity to this axis and biases the perceived color and lightness of test stimuli toward the orthogonal axis (unfilled circles and triangles). This causes achromatic increments to appear greenish and achromatic decrements to appear reddish and causes equiluminant reds to appear darker and equiluminant greens to appear brighter (unfilled triangles). The opposite color and lightness changes are induced by adaptation to a bright-green/dark-red modulation (filled triangles).

duced when observers adapt instead to the opposite pairing (bright green/dark red) of color and luminance (filled triangles). In all of these cases, the observed color and brightness changes reflect shifts away from the adapting color-luminance axis, consistent with a selective reduction in sensitivity to the adapting axis (Webster \& Mollon, 1991, 1994).

The biases in color appearance induced by contrast adaptation are analogous to the biases in the perceived orientation of lines in the tilt aftereffect (Gibson \& Radner, 1937) and, in fact, reflect "tilts" in the perceived directions of color space. Thus, adaptation to an oblique line ( bright red) causes a vertical line ( achromatic) to appear tilted in the opposite direction ( bright greenish). Similarly, in the ME, adaptation to the bright-red bars in a "red" grating may lead to a selective loss in sensitivity to bright red and thus tilt the appearance of an achromatic test grating toward bright green (Stromeyer \& Dawson, 1978; Webster, 1996). Equiluminant gratings may fail to induce an aftereffect in achromatic bars because they lie along a plane within color-luminance space that is orthogonal to the test grating (just as horizontal lines fail to induce a tilt aftereffect in vertical lines). However, adaptation to pure chromatic contrast does produce changes in the appear- ance of most other color-luminance directions, again by biasing their appearance away from the adapting color axis (Webster \& Mollon, 1994). And, in fact, MEs have been demonstrated previously for equiluminant adapting gratings when the aftereffects are measured with equiluminant test gratings rather than with the achromatic gratings used in conventional tests (Flanagan, Cavanagh, \& Crassini, 1989). For example, Flanagan et al. found that adaptation to equiluminant gratings, in which different color variations were presented at each orientation, caused the perceived hue of intermediate color directions to shift away from the adapting color directions at each orientation.

This account of the ME suggests that the specific pairings between luminance and color are as fundamental to the aftereffect as the pairings between orientation and color (Stromeyer \& Dawson, 1978; Webster, 1996). That is, the ME involves mechanisms that can respond selectively to different orientations, but the observed color changes reflect the changes in sensitivity to specific color-luminance combinations and not simply to the combination of orientation and color per se. In the present study, we explored how the ME depends on the relationships between color, luminance, and orientation within the stimuli. Our goal 

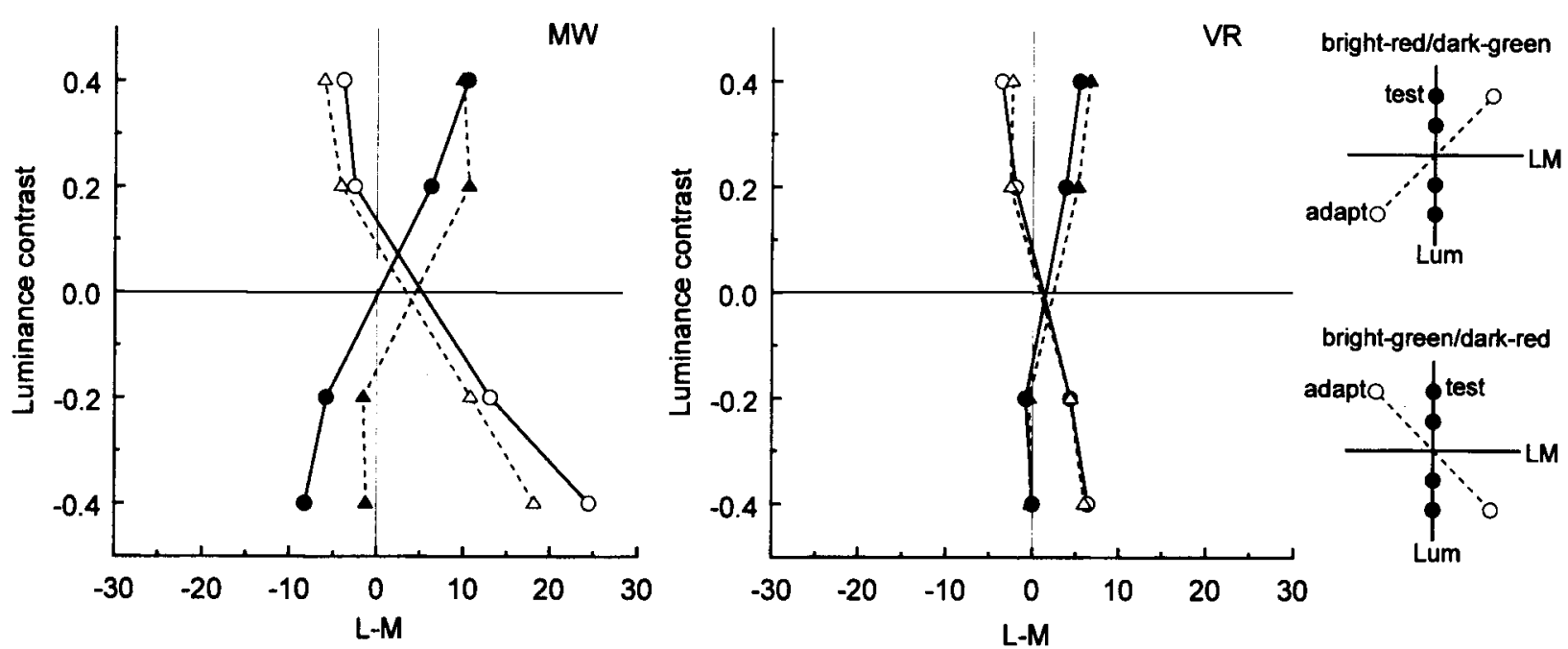

Figure 2. Perceived color changes in achromatic test gratings after adaptation to bidirectional gratings with alternating brightred/dark-green bars (unfilled symbols) presented at one orientation and bright-green/dark-red bars (filled symbols) at the second orientation (stimuli shown schematically in the color-luminance spaces to the right). Solid lines/circles plot the aftereffects for vertical gratings; dashed lines/triangles plot the aftereffects for horizontal gratings. [For reference, $L-M$ values of $-20,0$, and 20 correspond to CIE, $1931, x, y$ values of $(.295, .323),(.31, .316)$, and $(.324, .309)$ respectively.]

was to explore to what extent the color changes in the aftereffect can be predicted from biases against the colorluminance directions of the adapting gratings.

\section{METHOD}

\section{Apparatus}

Stimuli were presented on a computer-controlled color monitor. For most experiments, the display was a Sony 20SE monitor controlled by a Cambridge Research Systems VSG card. The experiments comparing the frequency dependence of the aftereffects were instead carried out on a BARCO CD351 controlled by a Sigma Graphics card. Both systems were calibrated to allow accurate specification of stimulus color and luminance on the display.

\section{Stimuli}

Except where noted, stimuli were horizontal or vertical squarewave gratings of 3.5 cycles/deg. In most conditions, the adapting gratings were presented in a $4^{\circ}$ square field. The test grating was presented in a $2^{\circ}$ square field, with the second orientation shown in the surrounding $4.1^{\circ}$ field. Viewing distance was $250 \mathrm{~cm}$. The gratings were surrounded by a $6.4^{\circ} \times 8.5^{\circ}$ uniform field of the same mean luminance $\left(15 \mathrm{~cd} / \mathrm{m}^{2}\right)$ and chromaticity (CIE 1931 $x, y=0.31,0.316$; equivalent to llluminant $C$ ). Luminance contrast in the patterns was defined by Michelson contrast. The chromatic contrast of the gratings was defined according to a scaled version of the MacLeodBoynton (MacLeod \& Boynton, 1979) chromaticity diagram and is related to their $r, b$ coordinates by the following equations:

$$
\begin{gathered}
\mathrm{L}-\mathrm{M} \text { contrast }=\left(r_{m b}-0.6568\right) * 1955 \\
\mathrm{~S}-(\mathrm{L}+\mathrm{M}) \text { contrast }=\left(b_{m b}-0.01825\right) * 5533 .
\end{gathered}
$$

The $\mathrm{L}-\mathrm{M}$ contrast corresponds to the opposing signals in the longand medium-wavelength-sensitive cones at constant luminance and varies in color appearance from reddish to bluish green. The $\mathrm{S}-(\mathrm{L}+\mathrm{M})$ contrast corresponds to $\mathrm{S}$ cone excitation (opposed by a combination of $\mathrm{L}$ and $\mathrm{M}$ cone signals) at constant luminance and varies in color appearance from purple to yellow-green. These axes were chosen because they appear central to the representation of color at early postreceptoral levels (Derrington, Krauskopf, \&
Lennie, 1984; Mollon, 1989; Webster, 1996) and because they isolate cone subsystems with very different spatial properties (see below). Equiluminance was established for individual observers by minimum-motion or flicker photometry. The two chromatic axes were empirically defined by methods we have described previously (Webster, K. K. De Valois, \& Switkes, 1990; Webster \& Mollon, 1994) and differed slightly from the nominal axes given in the transformations above. Chromatic contrast and luminance contrast were combined to form a variety of different adapting gratings, as described for specific experiments below. For all gratings, the luminance and chromatic contrasts were chosen so that the mean luminance and chromaticity for the pair always equaled the mean of the test gratings and the background. This ensured that color biases in the test stimuli were not due to chromatic adaptation to an average color bias in the adapting stimulus pair. (We assumed that local eye movements over the course of the adaptation period were sufficient to adapt observers to the space-averaged color and luminance of the gratings, which were alternated in orientation but fixed in phase.)

\section{Procedure}

Testing procedures were similar to those we have used previously to assess color contrast adaptation effects for uniform fields (Webster \& Mollon, 1994). These procedures involved regular alternation between test and adapting patterns and short delays between adapting and testing and thus differed from the testing sequence typical in many previous studies of the ME. The observers viewed the display in a dark room. Each run began with a 3-min adaptation period during which the vertical and horizontal adapting gratings were presented in alternation at a rate of $1 \mathrm{~Hz}$ (a fast rate chosen to minimize chromatic adaptation to the individual gratings). Following this initial exposure, the test stimulus was displayed for $5 \mathrm{sec}$, while the observers matched the perceived color of the test gratings by adjusting the color of either the central test grating or a uniform $1^{\circ}$ circular comparison field (with the same luminance as the test bars and shown $3^{\circ}$ to either side of the center of the test grating field). The matching color was varied using a button box that controlled the hue or saturation of the matching stimulus. The test presentation was in turn followed by $10 \mathrm{sec}$ of reexposure to the adapting gratings, in order to maintain a constant adaptation state during the course of the run. The sequence of testing and readaptation was repeated until 

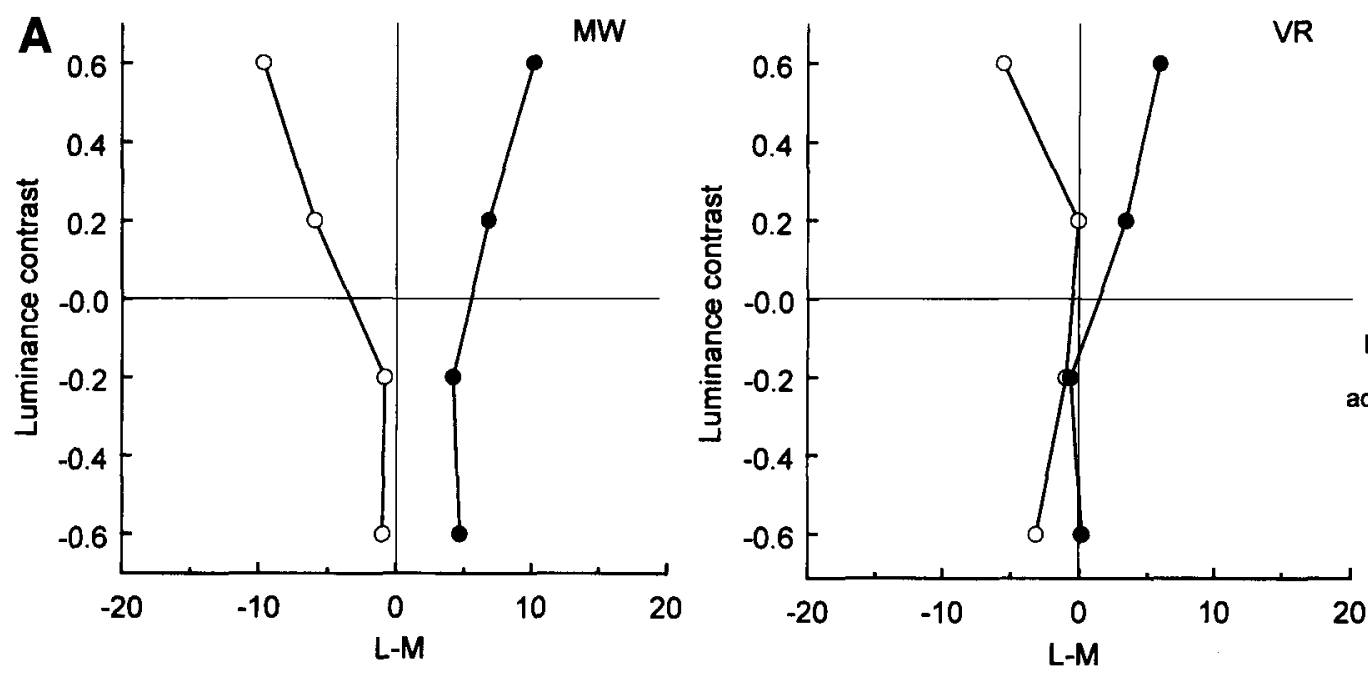

bright-red/dark-gray

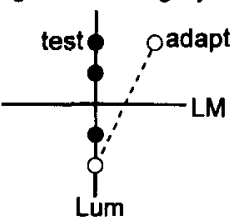

bright-green/dark-gray adapto dest
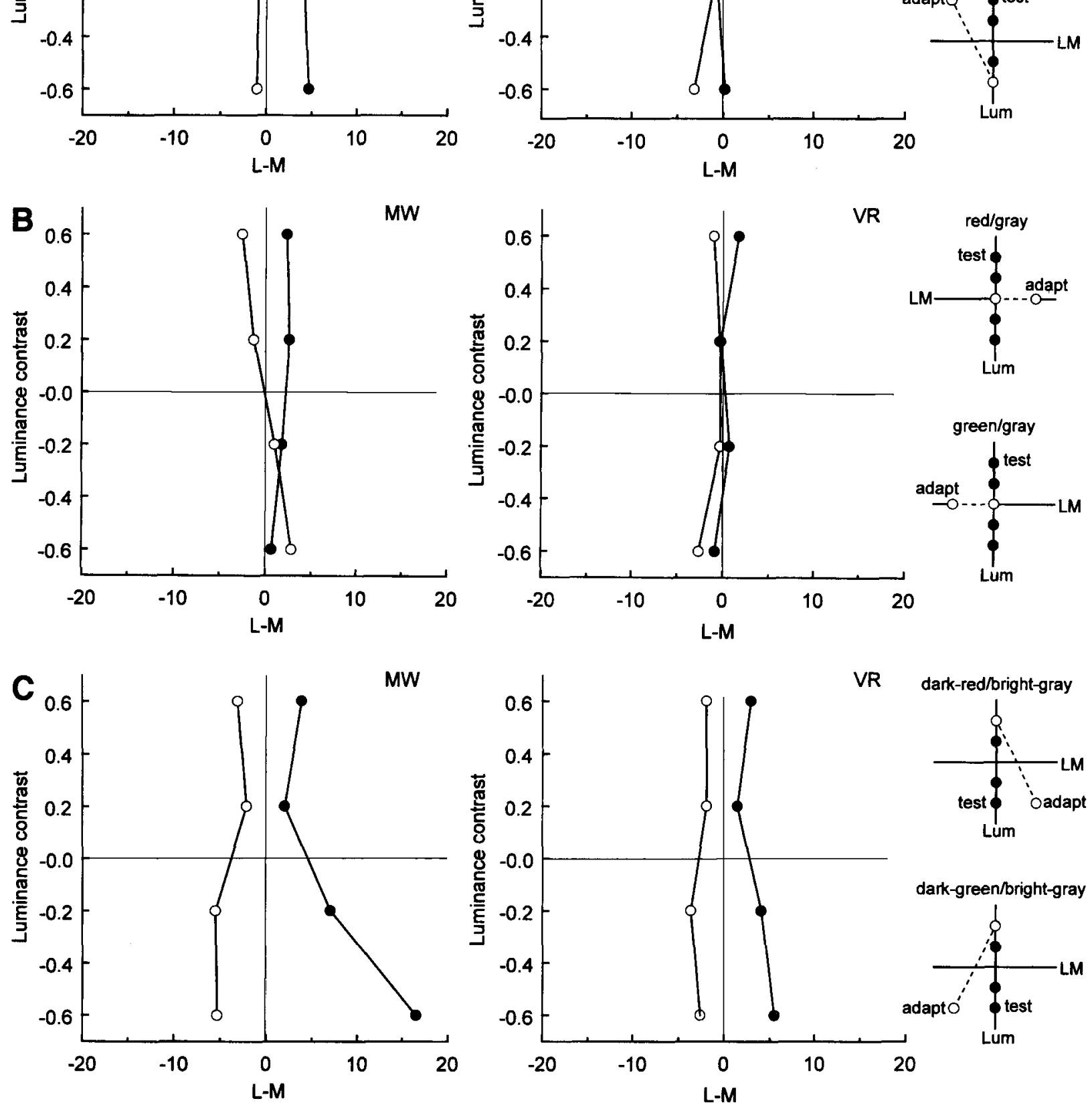

Figure 3. Perceived color changes in achromatic test gratings after adaptation to unidirectional gratings with alternating red (+L) and gray (achromatic) bars at one orientation (unfilled circles) and alternating green ( $-L$ ) and gray (achromatic) bars at the second orientation (filled circles) (see inserts). Aftereffects are shown after adaptation to bright chromatic/dark achromatic (A), equiluminant chromatic/achromatic (B), or dark chromatic/bright achromatic (C). 
the observers completed the match. Typically, six settings were made during a single adaptation run, with four to eight runs per session. Only a single adapting condition (color-luminance and orientation combination) was tested during any daily session, with the order of adapting and test conditions (and the pairing of color-luminance combination and orientation) counterbalanced across sessions. Points plotted are the average of four to eight settings per condition. The subjects included 1 author (M.W.) and 4 undergraduate observers who participated as paid observers or for course credit. All observers had normal color vision as assessed by standard color tests and normal or corrected-to-normal visual acuity.

\section{RESULTS}

\section{Bidirectional Color-Luminance Gratings}

In the first experiment, we tested for MEs in achromatic test gratings after adapting to pairs of gratings that had the same average color and luminance but differed in how color and luminance were combined. An ME in the test gratings would therefore have to be selective for the specific color-luminance combinations in the adapting gratings. The adapting bars alternated between bright red and dark green for one orientation and bright green and dark red for the second orientation (see Figure 2 inserts). The "red-green" difference was defined by a contrast of -75 or +75 along the $L-M$ axis, while luminance contrast was 0.4 . The test gratings were achromatic with a luminance contrast of 0.2 or 0.4 . Webster and Mollon (1993) have shown previously that adaptation to a single grating that covaries in luminance and color induces a perceived color change in achromatic gratings and a perceived brightness change in equiluminant test gratings. Here, we tested whether the perceived hue shifts exhibit the orientation selectivity characteristic of MEs.

Figure 2 shows measurements of the color biases induced in achromatic test gratings. The observers matched the apparent color of the bright or dark bars of the test grating by adjusting the $\mathrm{L}-\mathrm{M}$ contrast in the comparison field (which had the same luminance as the test bar). The results are plotted with test luminance contrast on the $y$ axis and matching $\mathrm{L}-\mathrm{M}$ chromatic contrast on the $x$-axis in order to represent the appearance of the patterns within the $\mathrm{L}-\mathrm{M}$ versus luminance plane. (For comparison, reference CIE values for the $\mathrm{L}-\mathrm{M}$ contrasts are noted in the legend.) Adaptation to the bright-red/dark-green gratings caused the bright bars of the test grating to appear greenish and the dark bars of the test grating to appear reddish. Opposite aftereffects were induced concurrently by the two different adapting orientations, which combined the same color and luminance components but in opposite ways. Moreover, the orientation dependence of the aftereffects reversed when the pairing of colorluminance direction and orientation was reversed. Thus, the hue shifts show clear orientation selectivity. (As Figure 2 shows, for a given color-luminance combination, the aftereffects were similar in form whether it was presented as the horizontal or vertical orientation. We have therefore averaged across orientation in subsequent figures.) The perceived color changes in both poles of the test gratings (bright and dark bars) represent rotations of the achromatic axis away from the direction of the adapting axis, as expected from a sensitivity loss that is selective for the specific color-luminance direction of the adapting axis at each orientation. The pattern of color aftereffects observed thus appears similar in form to the color changes induced by color contrast adaptation in uniform fields.

The hue shifts in the ME tend to be larger as the luminance contrast of the test gratings increases (Stromeyer, 1978). However, this contrast dependence appears different if one instead considers the aftereffect as a bias in the perceived color-luminance direction of the test stimulus. At higher luminance contrasts, more color would be required to maintain the same perceived angle, or colorluminance ratio, in the aftereffect. However, the actual rotations in the aftereffects may actually grow weaker with increasing contrast, consistent with the generally weaker effects in contrast adaptation when the ratio of test to adapting contrast increases (Blakemore, Muncey, \& Ridley, 1973; Georgeson, 1985; Webster, 1996). (We have not plotted the results in terms of color-luminance angle because we did not measure the changes in perceived luminance contrast of the test stimuli.)

\section{Unidirectional Color-Luminance Gratings}

The preceding results suggest that the orientationspecific aftereffects typical of MEs depend not on the pairing between orientation and color but on the pairing between orientation and color-luminance direction. To explore the effect of color-luminance direction more closely, we next examined the aftereffects when the luminance of a single chromatic component (e.g., red or green) was varied in the adapting grating. This mimics more closely the stimuli used in conventional studies of the $\mathrm{ME}$ and was chosen so that we could try to separate the influence of the different poles of a single color-luminance axis (e.g., bright red or dark green). The adapting gratings were composed of alternating chromatic and achromatic bars, with red $(+75 \mathrm{~L}-\mathrm{M})$ chromatic bars at one orientation and green $(-75 \mathrm{~L}-\mathrm{M})$ at the second orientation (see Figure 3 inserts). The luminance difference between the chromatic and achromatic bars was varied around a fixed mean luminance so that the gratings had a luminance contrast of +0.4 (chromatic bars brighter), -0.4 (chromatic bars darker), or 0 (chromatic and achromatic bars equiluminant). Test gratings were again achromatic, with a luminance contrast of 0.2 or 0.6 .

Figure 3 again shows the $L-M$ contrast matches made to the bright or dark bars of the test gratings after adaptation. The three panels for each of 2 observers (M.W. and V.R.) show matches for adapting gratings in which the chromatic bar was brighter, equiluminant with, or darker than the achromatic bar. There is little aftereffect when the adapting gratings are equiluminant, confirming that the induced color change in achromatic test bars requires adaptation to stimuli that combine luminance and chromatic contrast (Allan et al., 1997; Ellis, 1977; Mi- 

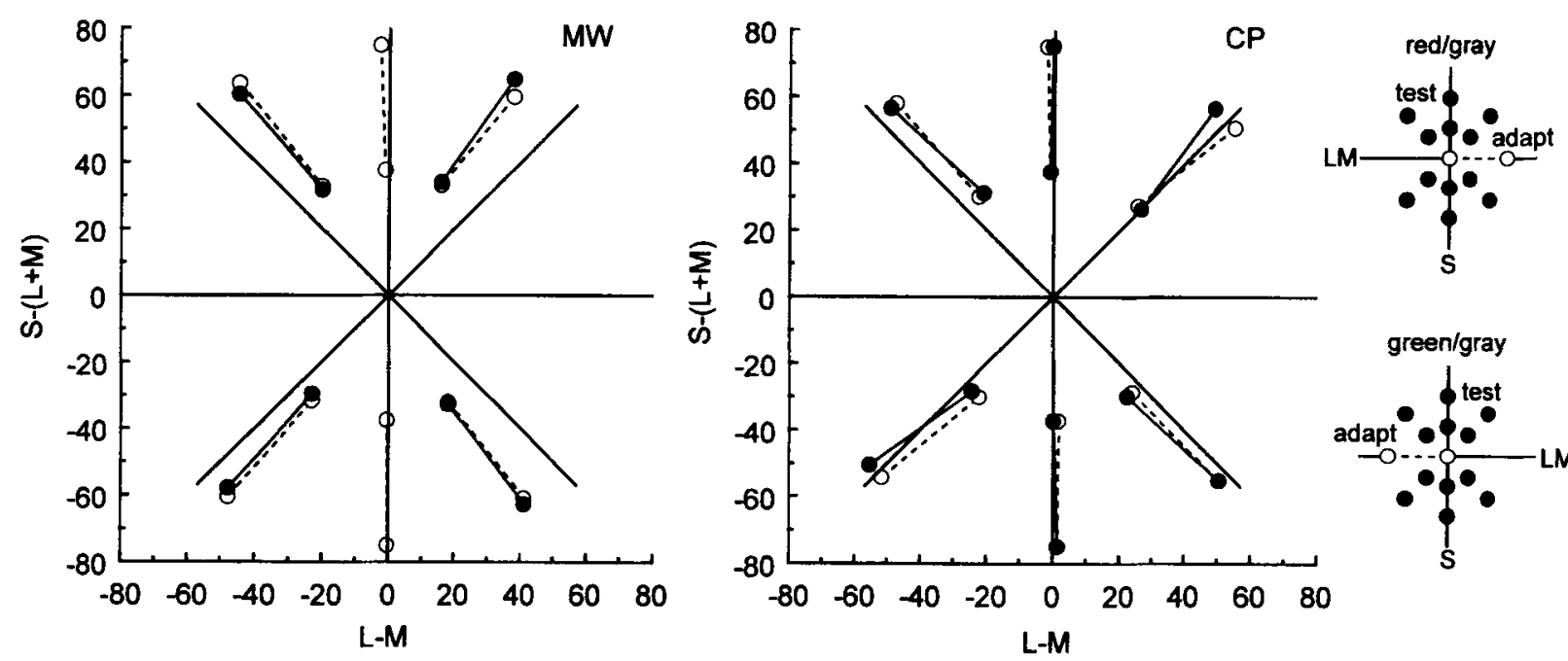

Figure 4. Matches in perceived hue angle of chromatic test gratings after adaptation to unidirectional, equiluminant red-gray gratings at one orientation (filled circles) and green-gray gratings at the second orientation (unfilled circles). Test gratings lay along chromatic axes of $45^{\circ}-225^{\circ}, 90^{\circ}-270^{\circ}$, or $135^{\circ}-315^{\circ}$ and had contrasts of 75 or 37.5 units in the $L-M$ versus $S-(L+M)$ plane (see inserts). The test gratings were composed of only one orientation, were shown above fixation, and were matched by adjusting the color angle of a comparison grating presented below fixation.

kaelian, 1980; Stromeyer \& Dawson, 1978). Alternatively, clear aftereffects were obtained when the chromatic adapting bars had either positive or negative luminance contrast. These aftereffects have two conspicuous features. First, the induced color shifts are in the same direction for both component bars of the test grating. That is, the dark-red adapting bars caused both the bright bars and the dark bars of the test to appear greenish, confirming previous studies (Mikaelian, 1980; Stromeyer, 1978). Thus, the pattern of aftereffects for the unidirectional gratings differ from the color changes for bidirectional gratings, in which opposite aftereffects were induced in the bright and dark bars of the test grating (Figure 2). Second, the magnitude of the aftereffects is stronger when the luminance contrast of the adapt and test stimuli have the same sign. That is, bright colored adapting bars induced larger effects in the bright bars of the test gratings than in the dark bars, and vice versa. This was verified with a twoway analysis of variance (ANOVA; test contrast $\times$ adapt contrast [color brighter vs. color darker], using the $a b$ solute value of the contrast matches at each orientation), which showed a significant interaction for both observers $[F \mathrm{~s}(3,88)=34.8$ and 8.0 for M.W. and V.R., respectively; $p<.001]$. The results are thus consistent with previous reports that the ME is selective for the luminance polarity of the gratings (Mikaelian, 1980; Stromeyer, 1978; Stromeyer \& Dawson, 1978). We consider the possible basis for these polarity-specific color shifts in the Discussion section.

\section{Equiluminant Adapting and Test Gratings}

As Figure 3 shows, color aftereffects in the achromatic test gratings were minimal when the unidirectional adapt- ing gratings were equiluminant. In the present measurements, we explored the basis for this absence by examining the pattern of aftereffects when the test gratings were also equiluminant. In the first case, the adapting gratings were again equiluminant unidirectional gratings (i.e., red-gray $[+75 \mathrm{~L}-\mathrm{M}]$ at one orientation and green-gray $[-75 \mathrm{~L}-\mathrm{M}]$ at the second orientation). The test gratings were bidirectional and composed of bars that alternated between either pole of the $S-(L+M)$ axis or intermediate axes of $45^{\circ}-225^{\circ}$ (reddish-purple-yellowish-green) or $135^{\circ}-315^{\circ}$ (bluish-yellowish), with a chromatic contrast of 37.5 or 75 units (see Figure 4 inserts). To facilitate matching the color of the test bars, we modified the display so that the adapting and test gratings (composed of a single orientation) were presented in a $2^{\circ}$ field centered $1.25^{\circ}$ above a small cross, which the observers fixated throughout. The perceived hue of the test bars was then matched by adjusting the (initially random) color angle of a comparison grating that was spatially identical to the test grating but presented in a $2^{\circ}$ field centered $1.25^{\circ}$ below fixation.

Figure 4 plots the coordinates of the hue matches within the $\mathrm{L}-\mathrm{M}$ and $\mathrm{S}-(\mathrm{L}+\mathrm{M})$ chromatic plane. Adaptation to the $\mathrm{L}-\mathrm{M}$ gratings had little effect on the perceived color of the test stimuli along the $\mathrm{S}-(\mathrm{L}+\mathrm{M})$ axis, which (like the achromatic axis defining the test gratings in Figure 3) is a direction in color-luminance space that is orthogonal to the $\mathrm{L}-\mathrm{M}$ chromatic axis and is thus a chromatic direction that should remain unbiased following L-M adaptation (Webster \& Mollon, 1994). On the other hand, the intermediate color directions are shifted away from the $\mathrm{L}-\mathrm{M}$ adapting axis and toward the $\mathrm{S}-(\mathrm{L}+\mathrm{M})$ axis (though, for Observer C.P., a systematic shift appears only along 

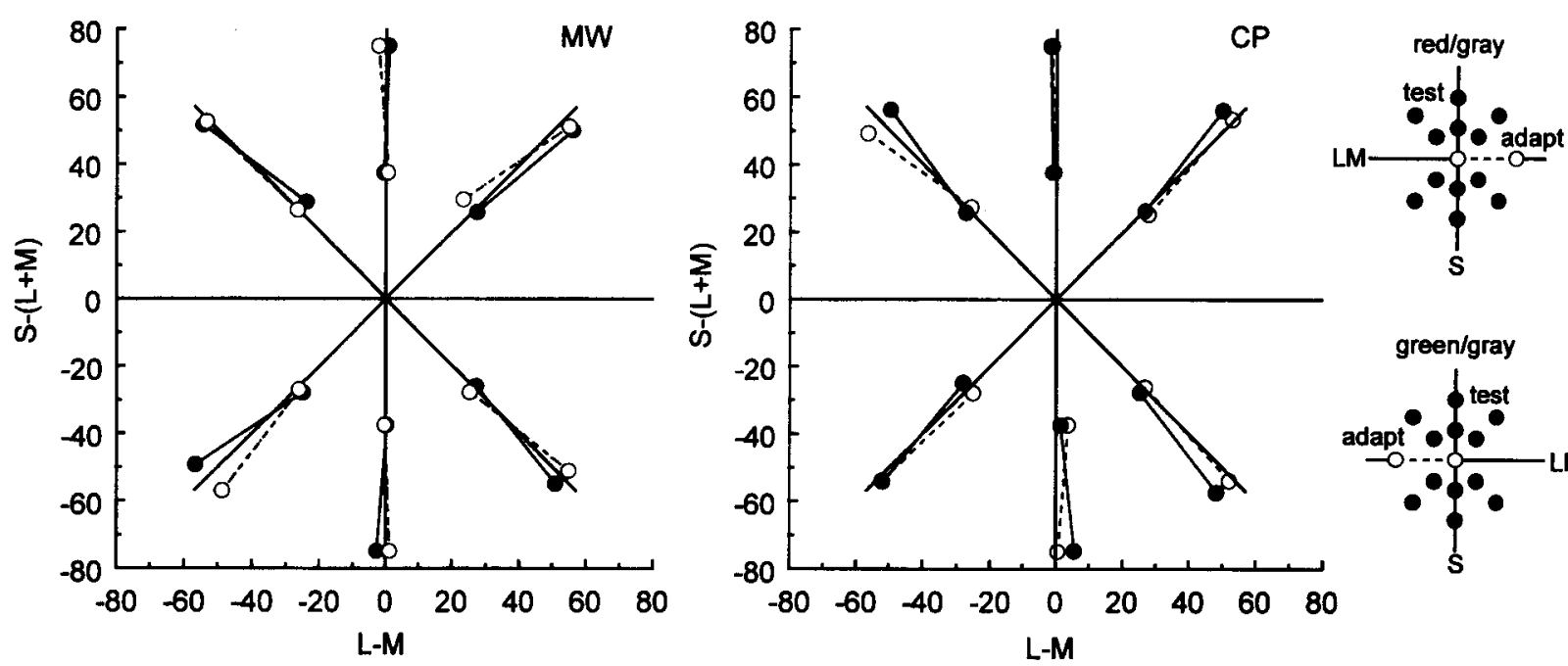

Figure 5. Cross-orientation matches in perceived hue angle of chromatic test gratings after adaptation to unidirectional, equiluminant red-gray gratings at one orientation (filled circles) and green-gray gratings at the second orientation (unfilled circles) (see inserts). In this case, matches were made by adjusting the hue angle of the central grating (at one orientation) to match the perceived hue of the surround grating (at the second orientation). Adaptation produced little change in the hue matches across the two test orientations.

the $135^{\circ}-315^{\circ}$ axis). These hue shifts suggest that the adapting gratings did induce a selective loss in sensitivity to the $\mathrm{L}-\mathrm{M}$ axis. However, the shifts appear similar for both adapting colors (and thus were similar for both test orientations), suggesting that this sensitivity loss was not orientation selective. To test for orientation selectivity more directly, we returned to the original stimulus configuration ( $4^{\circ}$ adapt and test fields that were centrally fix- ated, with both orientations present in the test pattern), and, this time, we measured the aftereffects by adjusting the color angle of the central test orientation to match the perceived hue of the surrounding orientation. Thus, in this case, the color matches should directly reflect the hue difference between the two test orientations, apart from any hue shifts that might be common to both orientations. The resulting matches are plotted in Figure 5 and show
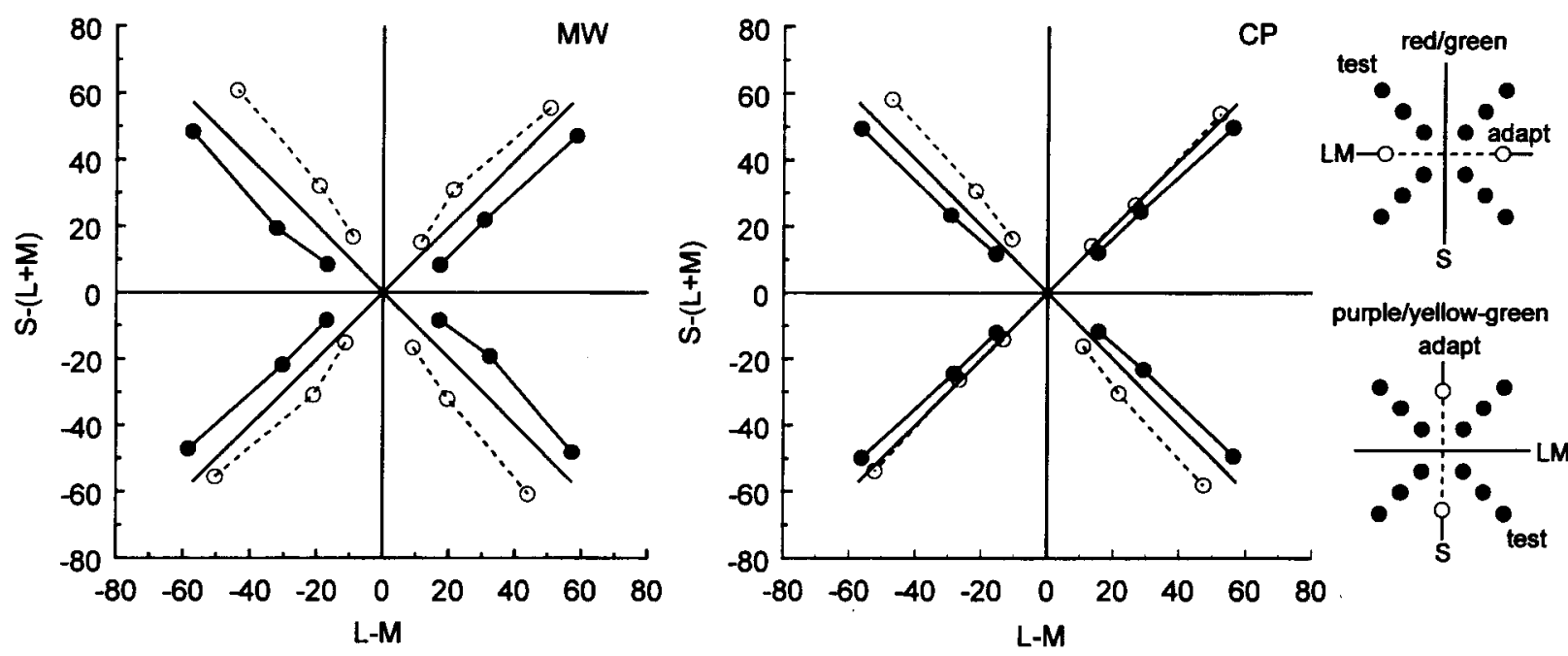

Figure 6. Matches in perceived hue angle of chromatic test gratings after adaptation to bidirectional, equiluminant gratings, with $\mathrm{L}-\mathrm{M}\left(0^{\circ}-180^{\circ}\right)$ gratings at one orientation and $S-(\mathrm{L}+\mathrm{M})\left(90^{\circ}-270^{\circ}\right)$ gratings at the second orientation. Test gratings lay along chromatic axes of $45^{\circ}-225^{\circ}$ or $135^{\circ}-315^{\circ}$ and had contrasts of $75,37.5$, or 19 units in the $L-M$ versus $S-(L+M)$ plane (see inserts). Test gratings that had the spatial orientation of the $L-M$ grating had to be rotated toward the $L-M$ axis to match gratings with the spatial orientation of the $S-(L+M)$ axis (filled circles), or vice versa (unfilled circles). This indicates that the perceived hue of the test gratings was biased away from the hue angle at each adapting orientation. (In this experiment, matches were not set separately for the two chromatic poles of the test grating. Points plotted have therefore been reflected across each axis.) 

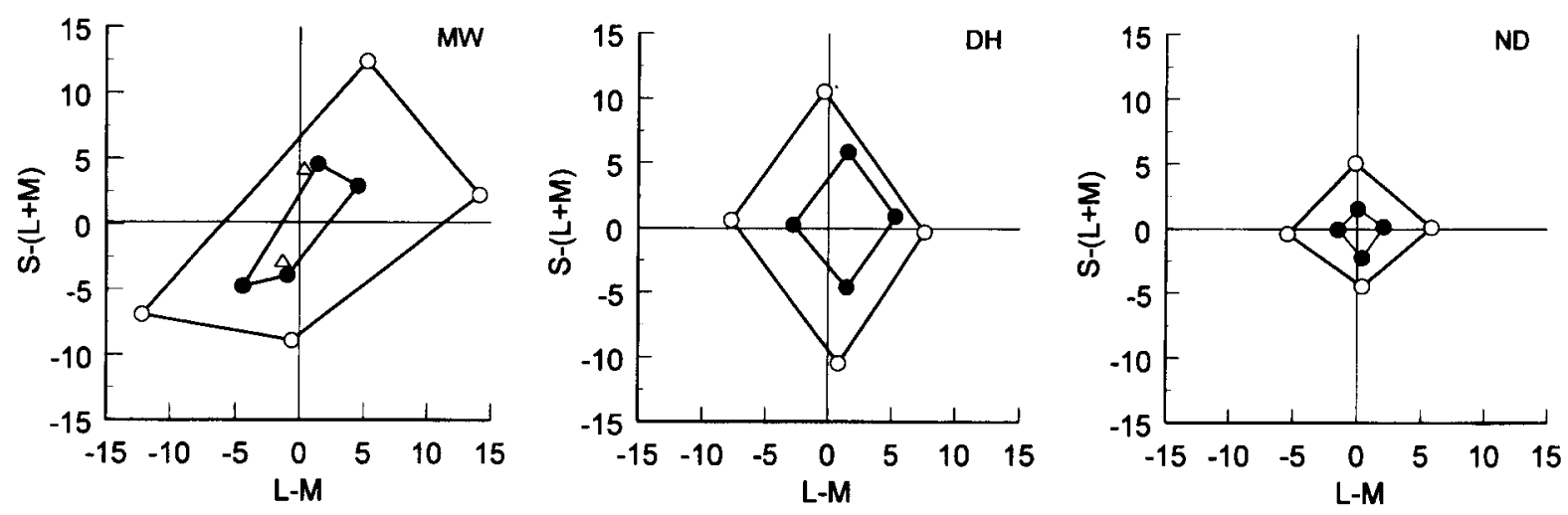

Figure 7. Matches to achromatic test gratings after adapting to $L-M$ gratings $(+L$ at one orientation and $-L$ at the second orientation) or $S-(L+M)$ gratings. Adapt and test gratings had a spatial frequency of 6 cycles/deg (unfilled circles) or 24 cycles/deg (filled circles). Matches were made by varying both the hue and the saturation of the comparison field. Unfilled triangles for M.W. show additional settings made after adapting to 24 cycles/deg Gabor patches instead of squarewave gratings.

no consistent rotation in the perceived direction of the test stimuli. Thus, this confirmed that the color changes induced in intermediate color angles by the red-gray and green-gray adapting pair (as revealed in Figure 4) were not orientation selective (i.e., the color shifts at both orientations were in the same direction).

As a second test of equiluminant MEs, we examined the aftereffects for bidirectional equiluminant gratings, using stimuli similar to those reported by Flanagan et al. (1989). These adapting stimuli differed from the adapting pair above because now each orientation was defined by a different, orthogonal color axis rather than by opposite poles of the same axis. The observers adapted to an $\mathrm{L}-\mathrm{M}$ grating at one orientation and an $\mathrm{S}-(\mathrm{L}+\mathrm{M})$ grating at the second orientation and again adjusted the hue angle of the central test grating to match the hue angle of the surround grating. The test gratings were equiluminant gratings along chromatic axes of $45^{\circ}-225^{\circ}$ or $135^{\circ}-315^{\circ}$ and had chromatic contrasts of $19,37.5$, or 75 units (see Figure 6 insert).

Figure 6 shows that, for these bidirectional adapting gratings, there are now systematic orientation-specific hue shifts in the test gratings. At each orientation, the intermediate test axes rotated in perceived color away from the color direction of the adapting orientation. Our results thus replicate the equiluminant color aftereffects reported by Flanagan et al. (1989). The absence of aftereffects at equiluminance in the conventional $\mathrm{ME}$ is therefore unlikely to reflect properties of spatial coding at equiluminance but instead may reflect properties of color coding - and, in particular, how the color of unidirectional gratings is encoded. Specifically, our results suggest that, at equiluminance, orientation-specific aftereffects occur when the two orientations are defined by different chromatic axes (as in the bidirectional gratings) but not when the two orientations are defined by different poles of a single chromatic axis (as in the unidirectional gratings we used). The lack of orientation selectivity for the unidirectional gratings suggests that in this case, the two opposite chromatic poles (at the two orientations) were both inducing the same-bidirectional-color shifts (i.e., both the red-gray and the green-gray gratings induced color changes consistent with red-green adaptation). In the Discussion section, we consider the possible bases for the different effects for unidirectional versus bidirectional chromatic gratings.

\section{McCollough Effects for $L-M$ or $S-(L+M)$ Axes}

In the final set of experiments, we compared conventional MEs for patterns defined by different chromatic axes. The $\mathrm{S}$ cones and their pathways differ in a number of ways from the $L$ and $M$ cones. For example, $S$ cones contribute little to conventional measures of luminance (Lennie, Pokorny, \& Smith, 1993). Moreover, because there are few $\mathrm{S}$ cones and they are sparsely distributed (Curcio et al., 1991; De Monasterio, McCrane, Newlander, \& Schein, 1985; Marc \& Sperling, 1977), the spatial resolution limit of the $\mathrm{S}$ cone mosaic is substantially lower than the L or M cone mosaic (Green, 1968; Stiles, 1949; Stromeyer, Kranda, \& Sternheim, 1978; Williams \& Collier, 1983; Williams, Sekiguchi, \& Brainard, 1993). We therefore asked whether these differences in spatial sensitivity would result in differences in the spatial dependence of the aftereffect.

To examine this, we compared the spatial frequency dependence of the ME for adapting colors that isolated the $\mathrm{L}-\mathrm{M}$ or $\mathrm{S}-(\mathrm{L}+\mathrm{M})$ axis. The adapting gratings were unidirectional and had a luminance contrast of 1.0 , and they were thus composed of alternate black and chromatic bars (e.g., of $+85 \mathrm{~L}-\mathrm{M}$ at one orientation and $-85 \mathrm{~L}-\mathrm{M}$ at the second orientation). Test gratings were achromatic bars with a luminance contrast of 1.0 . The adapt and test bars had a spatial frequency of 3 or 12 cycles/deg (pattern size of $2.6^{\circ}$ viewed from $370 \mathrm{~cm}$ ) or 6 or 24 cycles $/$ deg (pattern size of $1.3^{\circ}$ and viewed at $740 \mathrm{~cm}$ from the display through a front surface mirror).

Figure 7 plots the $\mathrm{L}-\mathrm{M}$ and $\mathrm{S}-(\mathrm{L}+\mathrm{M})$ coordinates of matches for the 6 or 24 cycles/deg gratings. The matches 

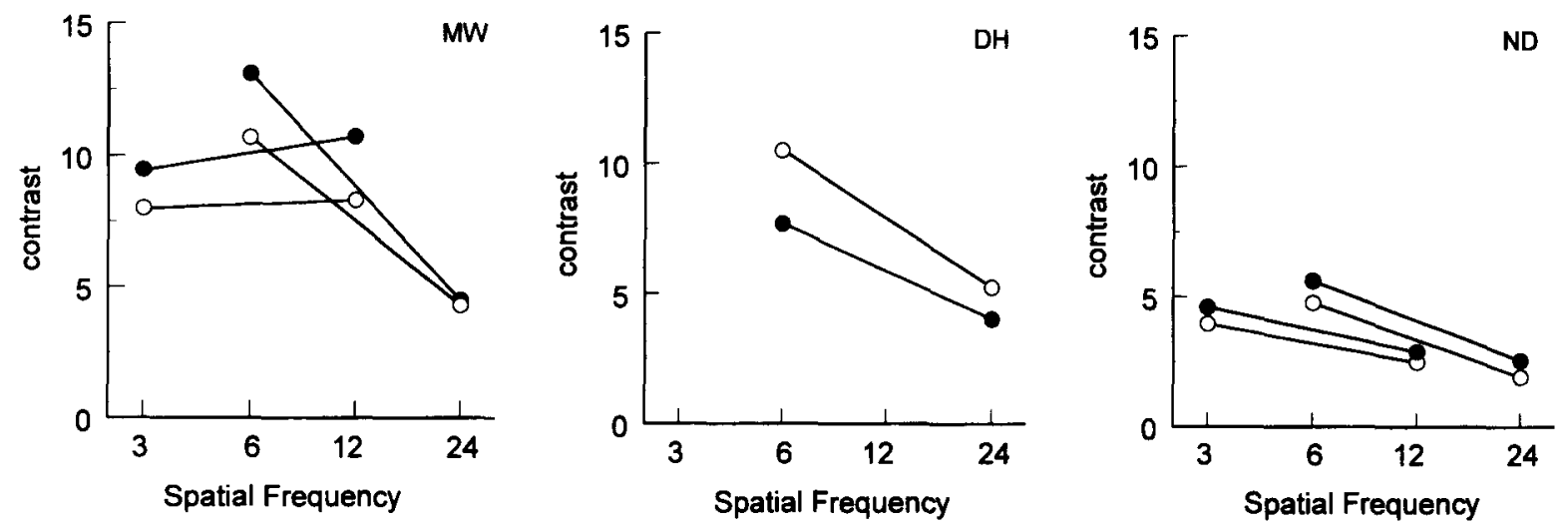

Figure 8. Magnitude of aftereffects for $L-M$ (filled circles) and $S-(L+M)$ (unfilled circles) adapting gratings as a function of spatial frequency. The magnitude is plotted as the perceived color shift in the direction complementary to the adapting color. The aftereffects for both color directions show a similar dependence on spatial frequency.

were made by adjusting both the hue and the saturation of the achromatic test grating after adapting to horizontal or vertical gratings defined by the complementary color pair along either chromatic axis (i.e., $+\mathrm{L}$ vs. $-\mathrm{L}$, or $+\mathrm{S}$ vs. - S). Aftereffects were weaker but still clearly visible at the high frequency and tended to be in color directions that were complementary to the adapting colors (though, for M.W., there are systematic rotations of the axes). Moreover, the frequency dependence of the aftereffect appears similar for both chromatic axes. This is shown in Figure 8, which replots the aftereffects to show the magnitude of the perceived color shifts along the color direction complementary to the adapting direction. The frequency dependence for the $\mathrm{L}-\mathrm{M}$ and $\mathrm{S}-(\mathrm{L}+\mathrm{M})$ axes was assessed for each observer by comparing the magnitude of the aftereffects using a two-way ANOVA (spatial frequency $X$ chromatic axis), which showed that the color $X$ frequency interaction was not significant $[F \mathrm{~s}(2,44)=1.5$, 0.2 , and 0.8 for M.W., D.H., and N.D., respectively]. Similar results were also obtained for gratings of 3 or $12 \mathrm{cy}-$ cles/deg, when the display was viewed directly at the closer distance (Figure 8). The higher frequencies should be near ( 12 cycles/deg) or well above $(24 \mathrm{cycles} / \mathrm{deg})$ the resolution limit of the S cones (Green, 1968; Stiles, 1949; Stromeyer et al., 1978; Williams \& Collier, 1983; Williams et al., 1993), yet MEs for these patterns were readily visible and varied with frequency in a similar way for the $L-M$ and $S-(L+M)$ axes.

To confirm that the aftereffects were not the result of possible edge artifacts in the stimuli, M.W. repeated the measurements at 24 cycles/deg using Gabor patches as the adapting stimuli. That is, the stimuli were, in this case, composed of sinewave gratings tapered by a Gaussian envelope. The unfilled triangles in Figure 7 show that the MEs remained similar to those induced by the untapered squarewave patterns. We also examined whether observers might be able to resolve the orientation of the high frequency patterns with their $S$ cones. To test this, the patterns were viewed in the presence of a superposed long-wavelength (Wratten 25) adapting background, chosen to desensitize the $L$ and $M$ cones so that the stimuli should be visible only to the $S$ cones. Under these conditions, the adapting patterns appeared as diffuse but spatially uniform patches. Forced-choice trials showed that the observers were unable to detect the orientation of the pattern, whereas thresholds for detecting the color of the patterns were only mildly elevated. Moreover, in the presence of the background, the observers could not perceive aliased patterns which might arise from the undersampling of the gratings by the $\mathrm{S}$ cones (Williams \& Collier, 1983) and could give rise to spurious oriented edges. Our results therefore suggest that a difference in $S$ cone excitation can induce an ME even when the spatial frequency of the patterns is too high to allow the $\mathrm{S}$ cones to resolve the orientation difference. Thus, the ME persists even when the cones encoding the pattern orientation are different from the cones encoding the pattern color.

\section{DISCUSSION}

\section{The McCollough Effect and Color Contrast Adaptation}

To summarize, we have examined properties of the ME within the context of the color changes induced by color contrast adaptation. Color-contingent aftereffects, such as the ME, involve interactions between color and form or movement and are important in demonstrating that the processes underlying the color aftereffects must be selective for the spatial and temporal properties of the stimulus. However, the perceived color changes in the aftereffect may reflect the selectivity for the color-luminance relationships in the adapting and test patterns. As illustrated in Figure 1, opposite color-aftereffects in bright and dark stimuli can be induced in uniform fields after adapting to a correlated variation in luminance and color (Webster \& Mollon, 1991, 1994), and MEs may be induced when both adapting orientations share a common color (Figure 2; Allan \& Siegel, 1997; MacKay \& MacKay, 
1977; but see Humphrey, Dodwell, \& Emerson, 1985). Thus, color aftereffects can persist under conditions that reduce the possible correlations between pattern and color, yet preserve the contingencies between luminance and color.

In the present study, we explored the functional similarities between color contrast adaptation and the ME by examining the orientation selectivity of the color adaptation. Our results show that the induced changes in the perceived color of gratings are selective for pattern orientation but that these spatially selective changes also depend fundamentally on how the stimuli are "oriented" within color-luminance space. Thus, opposite aftereffects are induced by horizontal and vertical gratings that have the same luminance and chromatic contrasts but combined in opposite ways (Figure 2). When the two adapting orientations have different mean colors, the aftereffects again depend on how those colors are combined with luminance (Figure 3). Finally, our results replicate previous studies showing that equiluminant adapting patterns do not induce aftereffects in achromatic gratings, yet can, under appropriate conditions, induce orientationspecific biases in the perceived color of equiluminant test patterns (Figure 6). The interactions between the adapt and test patterns thus depend in systematic ways on the relationships between the color-luminance directions defining the stimuli.

That MEs might be related to other classes of patternselective adaptation effects is well recognized (e.g., Barlow, 1990; Stromeyer, Lange, \& Ganz, 1973). On the other hand, it is also likely that the ME and other contingent aftereffects include processes that are distinct from the processes underlying the contrast-sensitivity changes observed with adaptation to gratings or uniform fields. For example, the $\mathrm{ME}$ is known to have a very long persistence and to exhibit little interocular transfer (Harris, 1980; Stromeyer, 1978) and may include processes that are specifically involved in discounting color fringes at edges (Broerse, Vladusich, \& O'Shea, 1999; McCollough, 1965). Such features may distinguish the ME from other examples of contrast or pattern aftereffects. We did not use the long delays between adaptation and testing that are typical in studies of the ME, and the persistence of our color aftereffects remains untested. However, the colorluminance relationships revealed by our results appear similar in pattern to those suggested by previous measurements of the ME. This functional similarity suggests that it may be possible to account for the general nature of the color changes in different cortical aftereffects within a common framework that is focused on how the visual system is organized to represent information about color and luminance.

An important implication of this framework is that chromatic contrast and achromatic contrast, or different types of chromatic contrast, are not encoded by separate channels that adapt independently. Instead, the adaptation effects imply channels that can respond selectively to specific combinations of luminance and chromatic contrast
(Webster, 1996). This was suggested previously for the ME by Stromeyer and Dawson (1978) to account for their finding that the MEs are selective for the luminance polarity of the adapting gratings, which our present results confirm. By such accounts, the achromatic axis-which, at postreceptoral levels, is typically assumed to reflect an absence of activity within chromatic channels - corresponds instead to the distribution of activity across different color-luminance mechanisms. The color aftereffects induced in achromatic gratings may thus arise when this distribution is biased by selective adaptation to specific color-luminance directions.

\section{S Cones and the McCollough Effect}

We observed qualitatively similar color aftereffects whether the observers adapted to pattern colors that varied only in S cone excitation or only in $\mathrm{L}$ and $\mathrm{M}$ cone excitation. This result is notable in two regards. First, as we pointed out previously, $S$ cones are thought to contribute little to measures of luminance sensitivity (Lennie et al., 1993), yet we have argued that the basis for the color changes in the ME is the interaction between luminance contrast and chromatic contrast. However, it is likely that, in the ME, what is actually being biased is the perception of surface color and lightness, which depends on different visual subsystems (e.g., the P pathway) than the pathways isolated by standard luminance measurements (e.g., the M pathway). Webster and Mollon (1993) showed that adaptation-dependent interactions between color and luminance are weak or absent in tasks that directly measure luminance sensitivity (e.g., flicker photometry), whereas they are strong in tasks that instead depend on judgments of the apparent color and brightness (or lightness) of stimuli (Webster \& Mollon, 1993, 1994). The present results are consistent with our previous work showing that, in color appearance judgments, adaptation can be strongly selective for how $\mathrm{S}$ cone signals are combined with luminance contrast.

The second notable feature of our results is that the $S$ cone aftereffects showed a similar spatial frequency dependence to the $\mathrm{L}$ and $\mathrm{M}$ cone adapting colors and remained clearly visible at frequencies above the resolution limit of the $S$ cones (Figures 7 and 8). Again, for such stimuli, different cone types must have encoded the color ( $S$ cones) and orientation ( $L$ and $M$ cones) of the adapting and test stimuli. This result is reminiscent of the observation that an ME induced under photopic conditions can be visible under scotopic conditions (Stromeyer, 1974). The transfer of the aftereffect across illumination levels presumably reflects the convergence of rods and cones onto common pathways. In the present case, the results imply that chromatic information from $\mathrm{S}$ cones can "converge" with luminance and high-frequency spatial information from $L$ and $M$ cones. Whether this convergence reflects hard-wired mechanisms or soft-wired associations remains unknown, as more generally do the mechanisms underlying the ME (Barlow, 1990; Dodwell \& Humphrey, 1990; McCollough, 1965; Riggs, White, \& 

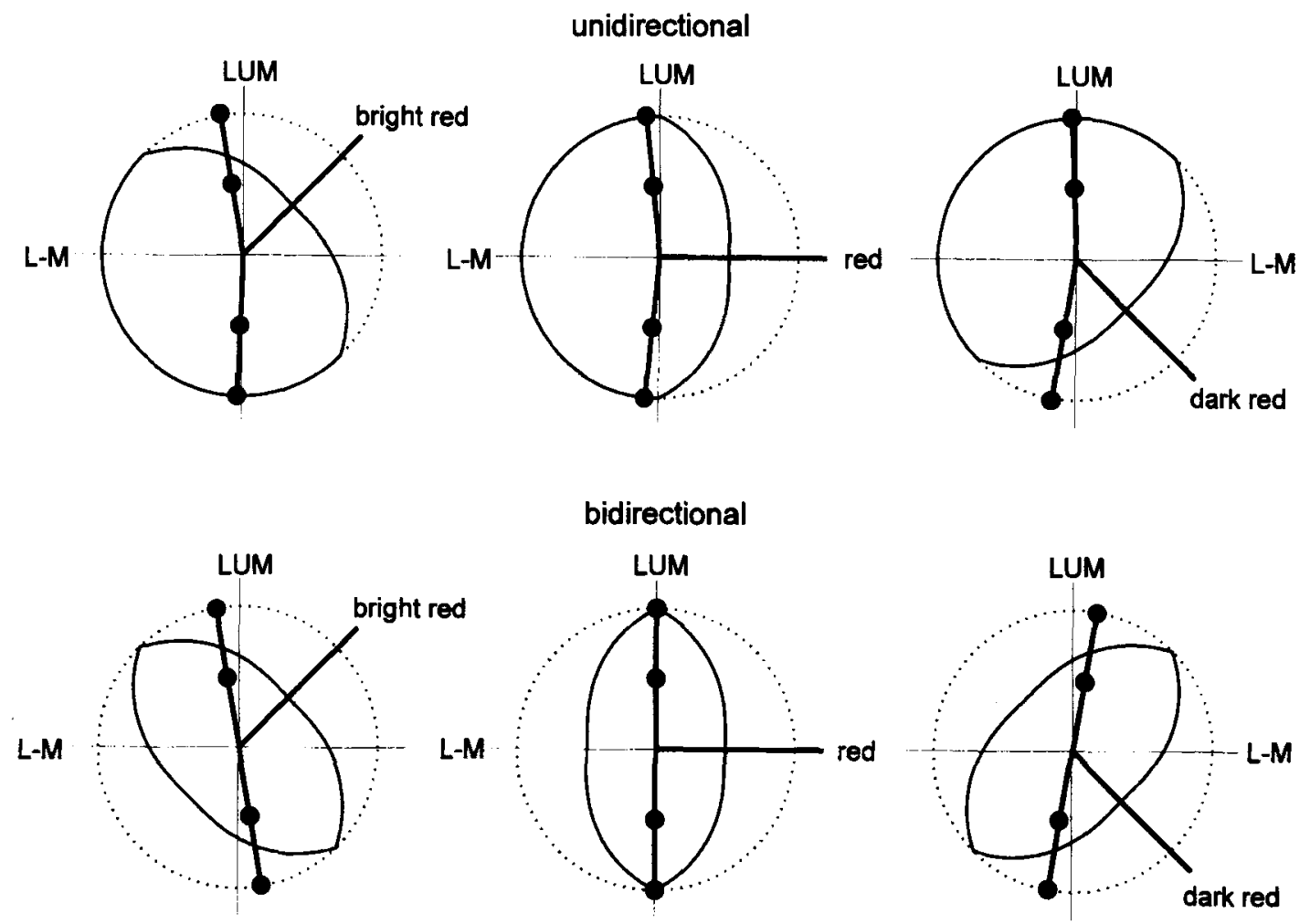

Figure 9. Perceived hue shifts in achromatic increments or decrements along the achromatic axis (filled circles) predicted by selective adaptation within unidirectional (top row) or bidirectional (bottom row) mechanisms tuned to different color-luminance combinations. Each individual mechanism has a sensitivity that varies as the cosine of its preferred color-luminance direction. The preferred directions of different mechanisms are assumed to be uniformly distributed around the luminance versus $L-M$ plane. The perceived direction of stimuli is assumed to correspond to the vector average of the population. Adaptation biases this average by reducing sensitivity within different mechanisms in proportion to their sensitivity to the adapting stimulus. Solid line contours plot the sensitivity to different color-luminance directions following adaptation to the adapting color-luminance axis. Adaptation in unidirectional mechanisms predicts hue shifts in the same direction in increments and decrements and predicts hue shifts frem equiluminant adaptation. Adaptation in bidirectional mechanisms predicts hue shifts in opposite directions in increments and decrements and predicts no hue shift from equiluminant adaptation.

Eimas, 1974; Siegel \& Allan, 1992). In either case, these results reveal an intriguing flexibility in the contingencies to which the visual system can adapt.

\section{Bidirectional Versus Unidirectional Color-Luminance Mechanisms}

We measured the color aftereffects for a variety of different adapting gratings that were either bidirectional or unidirectional--or, in other words, that included both poles or only a single pole of a color-luminance axis. What do the aftereffects for these stimuli imply about the polarity selectivity of the underlying mechanisms? To examine this, in this section, we compare our results to the color changes predicted by adaptation within mechanisms that have bidirectional or unidirectional spectral sensitivities. Conventional color-opponent channels are bidirectional in that they respond in antagonistic fashion to both poles of a color axis (e.g., exciting to red while inhibiting to green). Such channels were inspired by the mutually exclusive nature of color sensations (e.g., red vs. green; Hering, 1964) and describe how chromatic stimuli modulate the activity of geniculate cells (R. L. De Valois, Abramov, \& Jacobs, 1966). However, in many contexts, color mechanisms instead appear to be unidirectional, so that they are sensitive to excursions along only one direction of a color axis (e.g., either red or green). Unidirectional mechanisms are in fact widely assumed in the separate on- and off-pathways of luminance processing (Fiorentini, Baumgartner, Magnussen, Schiller, \& Thomas, 1990). A similar polarity-specific organization within chromatic mechanisms has been deduced from asymmetries in color perception (Abramov, Gordon, \& Chan, 1991; R. L. De Valois, K. K. De Valois, Switkes, \& Mahon, 1997; Stromeyer, Lee, \& Eskew, 1992), and this organization might naturally arise from the half-wave rectification in cortical cells (R. L. De Valois \& K. K. De Valois, 1993; Livingstone \& Hubel, 1988). Moreover, previous studies have shown that, with appropriate stimuli, the two poles of the luminance or chromatic axes can be adapted independently (Beer \& MacLeod, 
1999; K. K. De Valois, 1977; Krauskopf, 1980; Krauskopf, Williams, \& Heeley, 1982; Krauskopf \& Zaidi, 1986). We asked whether the pattern of color changes in our adaptation results is consistent with unidirectional or bidirectional mechanisms.

Figure 9 shows the sensitivity changes predicted by adaptation to a bright-red, equiluminant-red, or dark-red adapting stimulus and how these bias the perceived color-luminance direction of stimuli along the achromatic axis (filled circles). In each case, we assumed that the luminance-chromatic plane is spanned by multiple mechanisms tuned to different color-luminance directions (so that each stimulus is encoded by a distribution of channels with overlapping sensitivities) and that the appearance of the stimulus is determined by the distribution mean. We also assumed that each individual mechanism combines the cone signals linearly, so that sensitivity varies as the cosine of its preferred direction. The model is analogous to multiple-channel models of the tilt aftereffect and similar to the multiple-channel model of color contrast adaptation described by Webster and Mollon (1994).

The top row of Figure 9 illustrates the aftereffects predicted for unidirectional mechanisms. Adaptation to the bright-red axis (e.g., from a grating formed by gray and bright-red bars) desensitizes any mechanisms responsive to this axis. This will produce the largest sensitivity change in the mechanism tuned to the adapting axis and progressively weaker changes up to $\pm 90^{\circ}$ from the adapting axis (as shown by the solid line contour, which plots the sensitivity to different color-luminance directions following the adaptation). In turn, this will change the distribution of responses encoding the achromatic test bars. (Note this distribution includes all mechanisms tuned to directions within $\pm 90^{\circ}$ of the achromatic axis.) The bright achromatic bars show a large bias toward green, since the "bright-red" mechanisms contributing to this axis have been strongly desensitized. However, the dark chromatic bars are also predicted to show a weaker shift toward green, since the "dark-red" mechanisms contributing to this axis have also been weakly desensitized. More generally, all directions within the plane should appear rotated away from the bright-red adapting axis and toward a dark-green axis $180^{\circ}$ away. When adaptation is instead to the dark-red axis, all test stimuli should appear rotated toward bright green, inducing a large green shift in the dark achromatic bars and a smaller shift in the bright achromatic bars. Finally, adaptation to the equiluminant red should bias appearance toward equiluminant green, predicting moderate green shifts of equal magnitude in both the bright bars and the dark bars. Thus, the important feature of the polarity-specific adaptation is that it predicts that adaptation to any reddish axis will shift achromatic stimuli toward green, and the magnitude of the shift is larger for the test axis that is closer to the adapting axis.

The bottom row of Figure 9 shows the corresponding predictions for adaptation within bidirectional mechanisms. In this case, the bright-red stimulus maximally desensitizes a mechanism that encodes both bright red and dark green, thus inducing symmetrical losses in sensitivity. Bright achromatic stimuli are again rotated toward green, because the "bright-red (and "dark-green") mechanisms contributing to them are selectively adapted. Yet dark achromatic stimuli are now shifted toward red, because of the selective bias against dark green. More generally, in this case, all directions should appear to rotate away from the bidirectional adapting axis and toward an axis $90^{\circ}$ away. Unlike the previous predictions for unidirectional adaptation, this predicts aftereffects of opposite sign and equal magnitude for the opposite poles of the achromatic axis and predicts no aftereffect on achromatic tests from the equiluminant red grating.

How do these predictions relate to the pattern of aftereffects we actually observed? The results for brightred/dark-green adaptation of Figure 2 are consistent with either model, since the adapting stimulus included both axis poles. However, for the unidirectional stimuli of Figure 3, the bright-chromatic (Figure 3A) or dark-chromatic (Figure $3 \mathrm{C}$ ) adapting conditions yield results that are similar to the unidirectional predictions and clearly differ from the bidirectional predictions. Specifically, both conditions induced polarity-specific shifts of the same sign in the bright and dark achromatic test bars, confirming previous reports (Mikaelian, 1980; Stromeyer, 1978). (Note that our bright-red gratings alternated between bright red and dark gray, so that both components should have led to adaptation; however, including a sensitivity loss to dark gray does not alter the direction of the predicitions.)

Adaptation within unidirectional mechanisms is also consistent with reports that strong MEs can be obtained in gratings formed by bright and dark bars of the same chromaticity (Ellis, 1977). Within bidirectional mechanisms, the bright and dark components of the gratings should lead to opposite sensitivity biases, canceling the aftereffect. Yet, within unidirectional mechanisms, the separate bright and dark adaptation effects should both induce biases toward similar directions (e.g., toward dark green or bright green, following adaptation to a brightred/dark-red grating). This should cause both the bright bars and the dark bars of an achromatic test to appear greenish, as observed. Thus, unidirectional adaptation appears better able to account for the aftereffects for gratings with combined luminance and chromatic contrast.

Surprisingly, however, the equiluminant adapting conditions instead follow the predictions for bidirectional adaptation. Thus, adaptation to the gray-red stimulus did not induce an aftereffect in the achromatic tests (Figure $3 \mathrm{~B}$ ) or in tests along orthogonal chromatic axes (Figures 4 and 5) but did alter the appearance of test axes that were less than $90^{\circ}$ from the adapting axis (Figures 4 and 6). Such aftereffects are inconsistent with a polarity-specific sensitivity loss and instead fit well with the predictions for bidirectional mechanisms.

We are therefore left with a marked asymmetry between the aftereffects for equiluminant and luminancechromatic gratings. While our results do not reveal the basis for this difference, there are a number of factors 
that could contribute. First, the mechanisms tuned to the equiluminant plane might show a weaker or different form of rectification. For example, Krauskopf et al. (1982) found that sawtooth modulation could adapt separately the two poles of either the bright-dark luminance axis or the red-green chromatic axis, yet the sign of the adapting phase differed for luminance and color. Moreover, Webster and Mollon (1994) found no difference between sawtooth and sinusoidal adaptation on the suprathreshold appearance of chromatic stimuli, with both causing comparable color changes along both poles of the adapting axis. Thus, the mechanisms tuned to the equiluminant plane may behave more like bidirectional mechanisms with regard to adaptation-induced changes in color appearance.

A second possibility is that chromatic adaptation and/or induction effects may effectively transform the equiluminant unidirectional stimulus into a bidirectional modulation. We chose a rapid rate of alternation $(1 \mathrm{~Hz})$ between the adapting gratings to try to maintain a constant state of chromatic adaptation. Yet any differential chromatic adaptation during the $0.5 \mathrm{sec}$ at each orientation would shift the effective chromatic modulation toward a bidirectional red-green. Similarly, any chromatic induction would shift the perceived color of the gray bars toward the complementary color of the chromatic bars, again resulting in a red-green modulation. Color induction may precede the site of contrast adaptation, observers can be adapted by an apparent color modulation of a field that is induced by physically modulating color in the surround (Krauskopf \& Zaidi, 1986). On the other hand, induced colors have not been found to be effective adapting stimuli in previous studies of the ME (Thompson \& Latchford, 1986; W. R. Webster, Day, \& Willenberg, 1988), so the role of induced colors in the present results remains uncertain. Nevertheless, we informally observed that effects of chromatic adaptation and/or induction were strong at equiluminance, since the achromatic bars in fact appeared saturated and complementary in color to the inducing bars. Thus, chromatic adaptation and induction may simply have foiled our attempt to produce a unidirectional stimulus.

A third possibility is that the ME with luminance gratings does depend on special processes specific for luminance contrast, which, again, might involve mechanisms for compensating for chromatic fringes at luminance edges (Broerse et al., 1999; McCollough, 1965). In this event, it is unlikely that the absence of the conventional $\mathrm{ME}$ at equiluminance results simply because equiluminant stimuli fail to induce orientation-specific color aftereffects. First, our results (Figure 6) and those of Flanagan et al. (1989) show that that the aftereffects are orientation selective for bidirectional adapting patterns. Second, measures of threshold adaptation (Bradley, Switkes, \& K. K. De Valois, 1988), the tilt aftereffect (Elsner, 1978; Flanagan, Cavanagh, \& Favreau, 1990), and near-threshold and suprathreshold discrimination (Webster et al., 1990) have all found strong orientation selectivity for chromatic patterns. Thus, the asymmetries in the aftereffects are more likely to reflect asymmetries in color-luminance coding than in spatial processing. And, despite such asymmetries, our results show that it is plausible to assume that the color biases in all color-luminance directions reflect a similar principle-that they are effectively tilt aftereffects in the perceived directions of color space.

\section{REFERENCES}

Abramov, I., Gordon, J., \& Chan, H. (1991). Color appearance in the peripheral retina: Effects of stimulus size. Journal of the Optical Society of America A, 8, 404-414.

AllaN, L. G., \& SiEgEL, S. (1997). Contingent color aftereffects: Reassessing old conclusions. Perception \& Psychophysics, 59, 129-141.

Allan, L. G., Siegel, S., Kulatunga-Moruzi, C., Eissenberg, T., \& Chapman, C. A. (1997). Isoluminance and contingent color aftereffects. Perception \& Psychophysics, 59, 1327-1334.

BARLOW, H. B. (1990). A theory about the functional role and synaptic mechanism of visual aftereffects. In C. Blakemore (Ed.), Vision: Coding and efficiency (pp. 363-375). Cambridge: Cambridge University Press.

BEER, R., \& MACLEOD, D. (1999). Polarity-specific contrast adaptation. Investigative Ophthalmology \& Visual Science, 40, S176.

Blakemore, C., MunCey, J. P. J., \& Ridley, R. M. (1973). Stimulus specificity in the human visual system. Vision Research, 13, 19151931 .

Bradley, A., Switkes, E., \& De Valois, K. K. (1988). Orientation and spatial frequency selectivity of adaptation to color and luminance gratings. Vision Research, 28, 841-856.

Broerse, J., Vladusich, T., \& O'Shea, R. P. (1999). Colour at edges and colour spreading in McCollough effects. Vision Research, 39, 1305-1320.

Curcio, C. A., Allen, K. A., Sloan, K. R., Lerea, C. L., Hurley, J. B., Klock, I. B., \& Milam, A. H. (1991). Distribution and morphology of human cone photoreceptors stained with anti-blue opsin. Journal of Comparative Neurology, 312, 610-624.

de Monasterio, F. M., McCrane, E. P., Newlander, J. K., \& Schein, S. J. (1985). Density profile of blue-sensitive cones along the horizontal meridian of macaque retina. Investigative Ophthalmology \& Visual Science, 26, 289-302.

Derrington, A. M., Krauskopf, J., \& Lennie, P. (1984). Chromatic mechanisms in lateral geniculate nucleus of macaque. Journal of Physiology, 357, 241-265.

DE VALOIS, K. K. (1977). Independence of black and white: Phase-specific adaptation. Vision Research, 17, 209-215.

De Valois, R. L., Abramov, I., \& Jacobs, G. H. (1966). Analysis of response patterns of LGN cells. Journal of the Optical Society of America, 56, 966-977.

De Valois, R. L., \& De Valois, K. K. (1993). A multi-stage color model. Vision Research, 33, 1053-1065.

De Valois, R. L., De Valois, K. K., Switkes, E., \& Mahon, L. (1997). Hue scaling of isoluminant and cone-specific lights. Vision Research, 37, 885-897.

Dodwell, P. C., \& Humphrey, G. K. (1990). A functional theory of the McCollough effect. Psychological Review, 97, 78-89.

ELLIS, S. R. (1977). Orientation selectivity of the McCollough effect: Analysis by equivalent contrast transformation. Perception \& Psychophysics, 22, 539-544.

ElSNER, A. (1978). Hue difference contours can be used in processing orientation information. Perception \& Psychophysics, 24, 451-456.

Fiorentini, A., Baumgartner, G., Magnussen, S., Schiller, P. H., \& ThOMAS, J. P. (1990). The perception of brightness and darkness: Relations to neuronal receptive fields. In L. Spillmann \& J. S. Werner (Eds.), Visual perception: The neurophysiological foundations (pp. 129-161). San Diego: Academic Press.

Flanagan, P., Cavanagh, P., \& Crassini, B. (1989). McCollough effects for equiluminant gratings. Investigative Ophthalmology \& Visual Science, 30(Suppl.), 130.

Flanagan, P., Cavanagh, P., \& Favreau, O. E. (1990). Independent 
orientation-selective mechanisms for the cardinal directions of color space. Vision Research, 30, 769-778.

GEORGESON, M. A. (1985). The effect of spatial adaptation on perceived contrast. Spatial Vision, 1, 103-112.

Gibson, J. J., \& RADNER, M. (1937). Adaptation, aftereffect and contrast in the perception of tilted lines: I. Quantitative studies. Journal of Experimental Psychology, 20, 453-467.

GreEN, D. G. (1968). The contrast sensitivity of the color mechanisms of the human eye. Journal of Physiology, 196, 415-429.

HARRIS, C. S. (1980). Insight or out of sight? Two examples of perceptual plasticity in the human adult. In C. S. Harris (Ed.), Visual coding and adaptability (pp. 95-149). Hillsdale, NJ: Erlbaum.

Hering, E. (1964). Outines of a theory of the light sense (L. M. Hurvich \& D. Jameson, Trans.). Cambridge, MA: Harvard University Press. (Original work published 1920)

Humphrey, G. K., Dodwell, P. C., \& Emerson, V. F. (1985). The roles of pattern orthogonality and color contrast in the generation of patterncontingent color aftereffects. Perception \& Psychophysics, 38, 343353.

KrausKopf, J. (1980). Discrimination and detection of changes in luminance. Vision Research, 20, 671-677.

Krauskopf, J., Williams, D. R., \& Heeley, D. W. (1982). Cardinal directions of color space. Vision Research, 22, 1123-1131.

KRAUSKOPF, J., \& ZAIDI, Q. (1986). Induced desensitization. Vision Research, 26, 759-762.

Lennie, P., Pokorny, J., \& SMith, V. C. (1993). Luminance. Journal of the Optical Society of America A, 10, 1283-1293.

Livingstone, M., \& Hubel, D. (1988). Segregation of form, color, movement, and depth: Anatomy, physiology, and perception. Science, 240, 740-749.

MACKAY, V., \& MACKAY, D. M. (1977). Multiple orientation-contingent chromatic aftereffects. Quarterly Journal of Experimental Psychology, 29, 203-218.

MacLeod, D. I. A., \& Boynton, R. M. (1979). Chromaticity diagram showing cone excitation by stimuli of equal luminance. Journal of the Optical Society America, 69, $1183-1186$.

MarC, R., \& SPERLING, H. (1977). Chromatic organization of primate cones. Science, 196, 454-456.

McCollough, C. (1965). Color adaptation of edge-detectors in the human visual system. Science, 149, 1115-1116.

MikaELIAN, H. H. (1980). Effective luminance contrast as a parameter in contingent aftereffects. Perception \& Psychophysics, 27, 531-536.

Mollon, J. D. (1989). “Tho' she kneel'd in that place where they grew..." Journal of Experimental Biology, 146, $21-38$.

Riggs, L. A., White, K. D., \& Eimas, P. D. (1974). Establishment and decay of orientation-contingent aftereffects of color. Perception \& Psychophysics, 16, 535-542.

SAvoY, R. L. (1987). Contingent aftereffects and isoluminance: Psy- chophysical evidence for separation of color, orientation, and motion. Computer Vision, Graphics, \& Image Processing, 37, 3-19.

Siegel, S., \& Allan, L. G. (1992). Pairings in learning and perception: Pavlovian conditioning and contingent aftereffects. In D. Medin (Ed.), The psychology of learning and motivation (Vol. 28, pp. 127. 160). New York: Academic Press.

STILES, W. S. (1949). Increment thresholds and the mechanisms of color vision. Documenta Ophthalmologica, 3, 138-165.

STROMEYer, C. F., III (1974). Form-specific colour aftereffects in scotopic illumination. Nature, 250, 266-268.

STROMEYER, C. F., III (1978). Form-color aftereffects in human vision. In R. Held, H. W. Leibowitz, \& H. L. Teuber (Eds.), Handbook of sensory physiology (Vol. 8, pp. 97-142). New York: Springer-Verlag. StromeYER, C. F., III, \& Dawson, B. M. (1978). Form-colour aftereffects: Selectivity to local luminance contrast. Perception, 7, 407-415.

Stromeyer, C. F., III, LANGe, A. F., \& GANZ, L. (1973). Spatial frequency phase effects in human vision. Vision Research, 13, 2345-2360.

Stromeyer, C. F., III, Lee, J., \& Eskew, R. T. (1992). Peripheral chromatic sensitivity for flashes: A post-receptoral red-green asymmetry. Vision Research, 32, 1865-1873.

Stromeyer, C. F., III, Kranda, K., \& Sternheim, C. E. (1978). Selective chromatic adaptation at different spatial frequencies. Vision Research, 18, 427-437.

THOMPSON, P., \& LATCHFORD, G. (1986). Colour-contingent aftereffects are really wavelength contingent. Nature, 320, 525-526.

WEBSTER, M. A. (1996). Human colour perception and its adaptation. Network: Computation in Neural Systems, 7, 587-634.

Webster, M. A., De Valois, K. K., \& Switkes, E. (1990). Orientation and spatial-frequency discrimination for luminance and chromatic gratings. Journal of the Optical Society of America A, 7, 1034-1049.

Werster, M. A., \& Mollon, J. D. (1991). Changes in colour appearance following post-receptoral adaptation. Nature, 349, 235-238.

WeBSter, M. A., \& Mollon, J. D. (1993). Contrast adaptation dissociates different measures of luminous efficiency. Journal of the $O p$ tical Society of America A, 10, 1332-1340.

Webster, M. A., \& Mollon, J. D. (1994). The influence of contrast adaptation on color appearance. Vision Research, 34, 1993-2020.

Webster, W. R., Day, R. H., \& WillenberG, K. (1988). Orientationcontingent color aftereffects are determined by real color, not induced color. Perception \& Psychophysics, 44, 43-49.

Williams, D. R., \& Collier, R. J. (1983). Consequences of spatial sampling by a human photoreceptor mosaic. Science, 221, 385-387.

Williams, D. R., Sekiguchi, N., \& Brainard, D. (1993). Color, contrast sensitivity, and the cone mosaic. Proceedings of the National Academy of Sciences, 90, 9770-9777.

(Manuscript received November 16, 1998; revision accepted for publication June 3, 1999.) 